\title{
Problematic Observations using Firefly Algorithm and its Variants
}

\author{
N. Venkata Ramana Gupta, Ramesh Makala
}

\begin{abstract}
Real world problems can be analyzed and solved effectively and efficiently by observing the environment and its phenomenon. We can analyze the nature of the problem by looking for a solution in the environment. The evolution of such systematic handling of the problematic scenario to find an amicable and efficient solution can be applied to real world physical problems. Thus the application of procedures (algorithms) by different species has evolved into a novel idea referred as Nature Inspired Algorithms. It has gained wider publicity and popularity in the present research. One of the most popular nature inspired algorithm which has grabbed the attention of the world is Firefly Algorithm (FA).The Firefly Algorithm has a wide range of applicability in different disciplines. We tried to compare this algorithm along with its variants. We also throw some light on various characteristics of the Firefly Algorithm that makes it a far more efficient algorithm when compared to the rest of such similar algorithms.
\end{abstract}

Keywords: Security, firefly, problem identification, problem solving, performance analysis.

\section{INTRODUCTION}

Xin-she Yang developed FA in 2007 and published about this algorithm in 2008[48, 49].The prominent characteristic of this Firefly algorithm called as mimicking or flashing lights. In mid months of a year open areas are the best places to sight Firefly in temperature regions. We can find nearly 2000 variants that express similar characteristic of mimicking flashes.

\section{The Performance of Firefly}

The Flashing depends on the type of species with each having an individual pattern and it depends on two fundamental functionalities like, to attract mating partners (communication) and another one is for prey [32].Flashing acts as a safeguard against other species and to stay away from them and acts as a warning for predators.

The Firefly has an efficient signaling system to communicate that includes flashing rhythmically, the flashing rate and time gap between successive flashes make the other species to come closer[32].

Some of the species use this pattern to attract the male species and become prey for these.

Revised Manuscript Received on December 30, 2019.

* Correspondence Author

N. Venkata Ramana Gupta*, Research scholar, Acharya Nagarjuna University, Guntur, Andhra Pradesh, India. Email: nallagattla@gmail.com

Dr. Ramesh Makala, Associate Professor, RVR \& JC College of Engineering, Guntur, Andhra Pradesh, India.

(c) The Authors. Published by Blue Eyes Intelligence Engineering and Sciences Publication (BEIESP). This is an open access article under the CC-BY-NC-ND license http://creativecommons.org/licenses/by-nc-nd/4.0/
The capability of the Firefly in synchronizing the flashes and their self-organized behavior makes them emerging. From the laws of physics we know that Intensity (I) of light is inversely proportional to the distance(r), given in a notation

$I \propto \frac{1}{r 2}$. The absorption co-efficient of the medium may make the intensity weaker. These two properties make light coming from Firefly visible up to a certain distance a few hundred meters which is enough for communication among the Fireflies. The light pattern can be used for the formulation of the objective function and for optimization. This generates a new optimization algorithms.

\section{Standard Firefly Algorithms}

We assume that any Firefly (FA) has 3 prominent characters

1. Unisex in nature : Each of the Fireflies are attracted to one another

2. Attractiveness: Attractiveness is proportional to brightness of each one of these moves towards much brighter Fireflies if no such comparative brightness the firefly will move randomly in search of brightest.

3. Objective function: The effectiveness, scope of the objective function are determined.

These algorithms are evaluated basing on the fitness function and mapped to the objective function which is attained through a maximization in terms of brightness. Depending on these three regulations the pseudo code for Firefly is

\section{Firefly Algorithm}

Objective function

$f(x), x=\left(x_{1}, \ldots, x_{d}\right) T$.

Generate an initial population of $n$ fireflies

$\boldsymbol{x}_{i}(i=1,2, \ldots, n)$.

Light intensity Ii at $\boldsymbol{x}_{i}$ is determined by $f\left(\boldsymbol{x}_{i}\right)$.

Define light absorption coefficient $\gamma$.

while $(t<$ MaxGeneration),

$$
\text { for } i=1: n \text { (all } n \text { fireflies) }
$$

for $j=1: n$ (all $n$ fireflies) (inner loop) if $(I i<I j)$

Move firefly $i$ towards $j$.

end if

Vary attractiveness with distance $r$ via exp $\left[-\gamma r^{2}\right]$.

Evaluate new solutions and

Update light intensity. end for $j$

end for $i$

Rank the fireflies and find the current global best $\boldsymbol{g}$ * end while

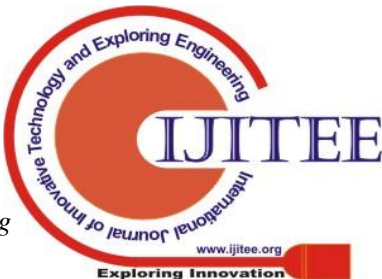


Post process results and visualization.

\section{Figure 1.1 Firefly algorithm (pseudo code)}

The basic architecture of Firefly is as mentioned in Figure 1.2

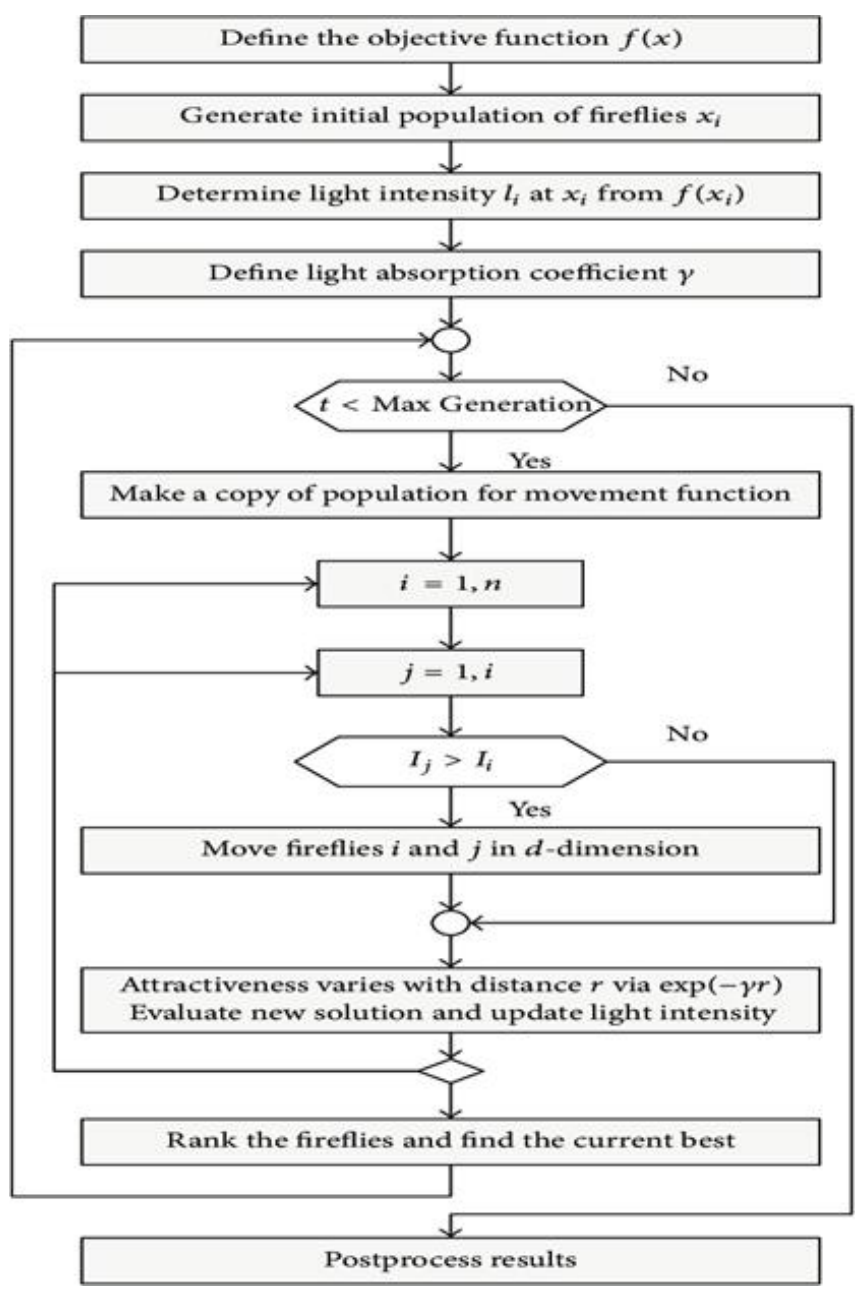

Figure 1.2: Firefly Architecture

\section{Variations in Intensity of light and measure of attractiveness}

The patterns are different for different species. The light intensity and attractiveness plays a significant role. The assumption that attractiveness is related to brightness and in turn related to objective function.

At a location ' $\mathrm{x}$ ' Firefly brightness ' $\mathrm{I}$ ' can be selected as $I(x) \propto f(x)$.

The parameter ' $\beta$ 'represents attractiveness. It is a subjective and relative attribute acts upon the individual Firefly. The absorption co-efficient or degree of absorption of media acts as an additive in formulating the attractiveness. The intensity of light varies with inverse square law

$I(r)=\frac{I s}{r 2}$

Where $I_{s}$ is source Intensity?

$\mathrm{r}$ - Distance

I - Intensity of Light

' $\gamma$ ' - Absorption co-efficient, Hence

$I=\begin{array}{lr}\mathrm{I}_{\mathrm{n}} \mathrm{e}^{-\gamma \mathrm{r}} & 1.2 \\ & I_{0} \text { Intensity of light at distance } \mathrm{r}=0 .\end{array}$
By combining Intensity and absorption basing on Gaussian law

$$
I(r)=I_{0} e^{-y r 2}
$$

From this above equation attractiveness $\beta$ can be estimated by

$$
\beta=\beta_{0} e^{-\gamma r 2}
$$

The above equation can be written as

$\beta=\frac{\beta 0}{1+y r^{2}}$

From eq. 1.4 and 1.5

The characteristic distance $\Gamma=\frac{1}{\sqrt{y}}$

Changes the attractiveness significantly from

$\beta_{0}$ to $\beta_{0} e^{-1}$

for eq 1.4

Or $\frac{\beta_{0}}{2}$

for eq 1.5

In general, monotonically decreasing can be

$\mathrm{B}(\mathrm{r})=\beta_{0} e^{\gamma r^{m}} \quad(\mathrm{~m} \geq 1)$

If absorption is constant the distinctive length is

$\Gamma=\gamma^{-\frac{1}{m}} \rightarrow 1 \quad(\mathrm{~m} \rightarrow \infty)$

Reversely for the problem of optimization usage of ' $\gamma$ ' is represented as

$\gamma=\frac{1}{\Gamma^{m}}$

The distance among two Fireflies is the Cartesian distance

$$
\begin{aligned}
r_{i j} & =\left\|x_{i}-x_{j}\right\| \\
& =\sqrt{\sum_{k=1}^{d}\left(x_{i k}-x_{j k}\right)^{2}}
\end{aligned}
$$

Can also be written as

$\left.r_{i j}=\sqrt{\left(x_{i}\right.}-x_{j}\right)^{2}+\left(y_{i}-y_{j}\right)^{2}$

The attractiveness can be given by

$$
x_{i}^{t+1}=x_{i}^{t}+\beta_{0} e^{-\gamma r 2 i j}\left(x_{j}^{t}-x_{i}^{t}\right)+\alpha \varepsilon_{i}^{t}
$$

The original expression is supplement with attractive and absorption co-efficient $2^{\text {nd }}$ and $3^{\text {rd }}$ terms.

' $\varepsilon_{i}$ ' Is a random number (Vector) drawn from a Gaussian distribution or uniform distribution.

\section{Randomization controlling}

In order to attain optima the algorithm has to converge along with this varying randomization parameter ' $\alpha$ ' has to decrease.

$\alpha=\alpha_{\infty}+\left(\alpha_{0}-\alpha_{\infty}\right) e^{-1}$

Where ' $\mathrm{t}$ ' $\in\left[0, t_{\max }\right]$ time used in simulations

$t_{\max }$ - The maximum no of generations

$\alpha_{0}$ - Initial randomization

$\alpha_{\infty}$ - is the final value.

As far as this algorithm of Firefly is considered use the global best $\left(g_{*}\right)$ in the final best solution computation other than using it explicitly.

To improve efficiency, we can add an additional term $\lambda \varepsilon \mathrm{i}\left(g_{*}-x_{i}\right)$ to the above 1.11 formula 
This ' $\lambda$ ' resemblances other ' $\alpha$ ' and ' $\beta$ ' and the vector ' $\varepsilon_{i}$ ' is a collection of random numbers.

From the 1.11 Equation random walk of Fireflies is more inclined in moving towards the brightness.' $\gamma$ ' parameter which is symbolizing the variation in attractiveness, it is more important in attaining convergence and behavior. The theory states $\gamma \varepsilon[0, \infty)$ but in practice optimization of the system with $\gamma=\mathrm{O}(1)$ determines the typical length ' $\Gamma$ ' and typical values range is 0.001 to 1000 .

\section{ANALYSIS OF THE ALGORITHMS}

The important characteristics are

\section{A. Scaling and limiting cases}

Basing on the type of the problem we are considering distance ' $r$ ' can be based on time for scheduling algorithms and it may be an amount of grouping and closeness of vertices. In optimization problems any quantitative representation of interesting term can be considered as ' $r$ ' the distance.

Assume that there are 'n' Fireflies which will be moving in randomly in the space basing on the brightness the Fireflies will be converging into local groups. The local optima converges into global optima.

Firefly Algorithm will reach global optima when $n \rightarrow \infty$ and $t>1$. Let ' $\Gamma$ ' is the scale of optimization for a huge number of Fireflies. i.e., $n>>\mathrm{k}$ where ' $\mathrm{k}$ ' is the local optima. The two limiting points are when $\gamma \rightarrow 0$ and $\gamma \rightarrow \infty$.

If $\gamma \rightarrow 0$ then $\beta=\beta_{0}$ (Constant attractiveness) then $\Gamma \rightarrow \infty$,

Absorption co-efficient is Zero (Ideal Condition)

Thus the flashing can occur globally and it leads to global optima basing on the algorithmic pseudo code eliminating the inner loop and substituting with present global best $\left(g_{*}\right)$.This becomes a variant of APSO and the efficiency is similar to PSO. The other side of this is $\gamma \rightarrow \infty$ Leads to $\Gamma \rightarrow 0$ and $\beta(r) \rightarrow \partial(r)$, it becomes a Dirac Delta function, attractiveness reaches to unmeasurable in the sight of other Fireflies. This is equal to the random roaming of fireflies in a region with foggy climate which becomes simulated Annealing (SA).Between these two opposite extremes, the firefly falling in between and varying ' $\gamma$ ' and ' $\alpha$ ' . Thus performance would outcome simulated Annealing( $\left.S_{*}\right)$ and PSO .We can find the global and local optima simultaneously and effectively. The independent nature reflects parallel implementation and aggregation that makes faster to find the convergent optimum.

\section{B. Attraction and Diffusion}

Assuming the light as a characteristic, attraction and intensity as parameters for exploitation was first used by Xin-she Yang in the FA in 2007 \& 2008.With the help of inverse square law, the light intensity called as attractiveness ، $\beta$ ' 0 'mapped with absorption coefficient ' $\gamma$ ' is represented as

$$
\boldsymbol{\beta}^{\left(-\boldsymbol{r}^{2}\right)} \text { Ata distance } \gamma=0 \text { and } \gamma>0 \text { as }
$$

Absorption co-efficient [48].

The attraction leads these independent systems to evolve, communicate, and correlate. This organized behavior enables a local optimality of the algorithm to converge rapidly towards the global optimal solution. Previously lot more algorithms have applied inverse square law by observing nature like a changed system search(CSS) used coulomb's law, law of gravitation was invented by newton applied in gravitational search algorithms(GSA).Attraction taken as a mechanism for the application on nature inspired computation and computational intelligence is a forerunner of its kind. The laws of fundamentals are same in view of meta-heuristics. The attraction factor made the agents to converge the swarm population. Through randomization and attraction both exploration and exploitation are possible. Consider here exploration in the sense of random walk and diffused chains. The random walk of an agent $\mathrm{x}_{\mathrm{i}}$, represented as

$$
x_{i}^{(\mathrm{t}+1)}=\mathrm{x}_{\mathrm{i}}^{(\mathrm{t})}+\varepsilon
$$

Where ' $\mathrm{t}$ ' is a counter of steps

' $\varepsilon$ ' is a random no of Gaussian normal mean distribution.

The average distance of an agent is the square root of a

finite no of steps ' $t$ '. Distance is $\sqrt{D t}$ where ' $\mathrm{D}$ ' is diffusion co-efficient .Moreover, the random walk in a ' $d$ '- dimensional case is

$$
\sigma^{2}(\mathrm{t})=\left|\mathrm{V}_{0}\right|^{2} \mathrm{t}^{2} \div(2 \mathrm{dD}) \mathrm{t}
$$

' $\mathrm{V}_{0}$ ' - is the velocity of drift $=0$ with respect to ' $\mathrm{t}$ ' . If it is proportionally large it can cover the entire search domain with a mean of zero and variance of time - dependent the Brownian $\mathrm{B}(\mathrm{t})$ obeys a Gaussian distribution. The process of diffusion can be treated as a series of Brownian motion that is in the form of Gaussian distribution .Hence it is referred as Gaussian diffusion, if the sequence is no Gaussian, it is referred as non-Gaussian. As random walk is by nature random it can take any form .Basing on the length of step it may take any other form of random walk. If the length of the steps is levy distribution, these are known as levy flights or levy walks. A combination of Firefly algorithm and levy flights gives a good performance [49].

\section{Special Cases of FA}

The prime characteristic of FA “attraction' is very strong in the local distance and its less in the longer distances. Hence the parameter itself subdivides the population into groups basing on the problem mode. Thus FA will deal with multi modal, non- linear optimization problems naturally. We can say basing on eq.1.11, If absorption co-efficient ' $\gamma$ ' is large the attraction rapidly decreases so the second part becomes ignorant. This makes it an SA. On the other side if ' $\gamma$ ' is very small (' $\gamma^{\prime} \rightarrow 0$ ) the power factor [- ' $\gamma^{\prime} \mathrm{r}^{2} \mathrm{i}$ j] $\rightarrow 1$ thus $x_{i}^{t+1}=x_{i}{ }^{t}+\beta_{0}\left(x_{j}{ }^{t}-\mathrm{x}_{\mathrm{i}}{ }^{t}\right)+\alpha \varepsilon_{\mathrm{i}}{ }^{t}$ 1.16 
This can transform into differential evolution if ' $\alpha$ ' $=0$.If the current global best replaces the term $x_{j}^{t}$, $x_{i}{ }^{\mathrm{t}+1}=\mathrm{x}_{\mathrm{i}}{ }^{t}+\beta_{0}\left(g_{*}-\mathrm{x}_{\mathrm{i}}{ }^{t}\right)_{+} \alpha \varepsilon_{\mathrm{i}}{ }^{t}$

Thus it becomes a APSO that was introduced by Xing She Yang in 2008[48].In eq 1.16 if $\quad \beta_{0}=0$ and $\varepsilon_{i}^{t}$ maps to $\mathrm{x}_{\mathrm{i}}$ , it becomes a harmony search(HS).All the DE,PSO, SA and HS are evolved from FA up to a certain extent.PSO and DE uses linear equations and FA has excellent behavior and great convergence as it uses non - linear equations .So, obviously FA outperforms other algorithms in a wide variety of applications like optimization, classification, image processing and feature selection.

\section{VARIANTS OF FIREFLY ALGORITHM}

\section{FA Variants}

To enhance convergence of FA a lot of experimentation was done by researchers in different ways. As a result new variants has been developed [19, 42, 37 ].The rapid expansion of literature is generating more variants some of them are listed as below.

1) Discrete Firefly algorithm (DFA) sayadi et.al [42] has applied FA to NP Hard scheduling problems and device a more suitable algorithms when compared with ACO. Durkota independently implemented DFA to AQP problems [14].When compared with otsu's and recursive otsu's methods, Hassanzad et.al [24] developed applied FA based method for image segmentation it is more efficient In solving NP hard traveling salesman problem[29] by Jatt and sujanto. The effectiveness of FA is clearly visible.The real coded FA is proposed to study constraint unit commitment problem [9] by Chandrasekhar and Simon.

2) Chaotic Firefly Algorithm (CFA) In Coelho et al. (2011) proposed chaotic FA, which is more superior to the rest [11, 12].

The performance of FA can be enhanced by varying ' $\beta$ ' and ' $\gamma$ ' parameters [51].Yang observed there intrinsic chaotic characteristics. By considering different chaotic maps company the performance showed that these can enhance the FA further by substituting some of the parameters with chaotic maps [21]

3) Lagrangian Firefly Algorithm (LFA). This was proposed by Rampriya et.al to solve unit commitment problem for a power systems[41]

4) Memetic Firefly Algorithm (MFA) To solve combinatorial graph coloring problems, Fister Jr. et.al applied this and attained better results [18].

5) Multi Objective Discrete Firefly Algorithm (MOFA) To study the economic emission load dispatch which was developed by Apostolopoulos and valcho, The comparative study resulted in stating that this is an efficient algorithm [6]. A new application area is grid computing where the consumption of the energy is minimized using the multi-objective FA(MOFA) in workload management tool for optimization by Arsuaga -Rio a nd Vega-Rodriguez[4].In

one more application this is used in production systems[33] by $\mathrm{Li}$ and Ye.

In another application a discrete FA variant is used for multi objective hybrid flow shop scheduling problem by Marichelvam et al [35]

6) Multi Objective Firefly algorithm (MOFA) The FA for single objective optimization is extended to multi objective optimization of continuous design problems by yang[53]

7) Multi Objective Enhanced Firefly Algorithm (MOEFA) For detection of the community in complex Networks, Multi Objective Enhanced FA is presented by Amiri et. al [3].

8) Hybrid Firefly algorithms (HFA) Due to its prominence the FA has been a hybridized with some more algorithms. The combination of FA with ant colony optimization by Finnakouris et.al has resulted in good optimization [22].The FA combined with differential evolution parameters of non linear biological model study resulted in a tool with a power of less consumption time for various applications [2].Even FA with adaptive strategies has been presented by Yan et al [55].

9) Parallel Firefly Algorithm with prediction (PFAP) The inverse heat conduction problems is handled with a parallel implementation of FA by Lvz et.al [34].

The development of discrete versions of FA have showed better performance for discrete problems and combinational optimization [42, 29, 18, 14 ]. These are applied in a wide variety of applications like traveling salesman problem, graph coloring etc. The investigation of extending FA to multi objective optimization even showed that chaos will improve the FA's performance [11, 51]. In other related studies the attempt to combine FA with this algorithm has been [22, 26, 27, 41].The newly improved or modified algorithm cannot be treated as new variants as there will be only slight modification to the already existing algorithms. The ' ' $\alpha$ ' called scaling factor has been obtained by replacing Gaussian distribution by uniform distribution by Farahani et.al with a little improvement[17].In UCAV path planning a new term discovery rate was added to FA by Wang et al[47] which indeed resulted in a better performance.

\section{HOW WE CAN DISCRETIZE FIREFLY ALGORITHM}

The development of FA has been taken place for the problem domains like continuous, multimodal optimization related fields, it can also be applied to ascertain combinatorial optimization related scenarios. To achieve this we have to know how to change variables of continuity into variable of discrete. It can be performed with the known sigmoidal logistic function

$$
s(x)=\frac{1}{1+\exp (-x)} 1.18
$$


This converts from continuous to a variable ' $\mathrm{S}$ ' of binary. The function for ' $\mathrm{x}$ ' gives $s \rightarrow 1$ as $x \rightarrow+\infty$ and $s \rightarrow 0$ as $x \rightarrow-\infty$

In practical situations this may not be an easy to attain as the right range of values might not be achieved. The combination of ' $r$ ' a random number along with the conditional switch That is

$$
(u>r) \rightarrow 1 \quad(\mathrm{u} \leq \mathrm{r}) \rightarrow 0
$$

When necessary we can modify $Y=2 S-1 \in\{+1,-1\}$

Since we can have $S \in\{0,1\}$

The deriving of $\mathrm{S}$ can be represented as

$$
\frac{d s}{d x}=\mathrm{s}(1-\mathrm{s})
$$

We can even add signs and randomization as $\mathrm{y}=\operatorname{sign}(\mathrm{x})$ for the generation range $+1,0$, -1 .Sometimes a simple way $y=$ $\lfloor x\rfloor$ a largest integer not greater than $\mathrm{x}$ even we can use a modulus function for example

$$
x \leftarrow\lfloor\mathrm{X}+\mathrm{k}\rfloor \bmod m, \quad \mathrm{k} \& \mathrm{~m}>0, \text { are integers. }
$$

One more dimension is how we can generate a latest solution as we have $s=+1$ or $s=-1$, Using these two with an increment/decrement of step at random time/iteration t we can have local random

$y^{t+1}=\mathrm{y}^{\mathrm{t}}+\mathrm{s}$

Basing on the design variables if they are binary the latest variables have to be normalized, a solution is to swap randomly within 0 \& 1 to have operations like mutations. In order to have a randomness use a random number with a discrete set. In a discrete search space ' $Z$ ' called a design variable will be mapped to a limited or finite set. Random number ' $u$ ' which distributes uniformly can be generated and we can map it to the cardinality of the set. The usage of finite set value is based on the drawn random number. The distance and neighborhood which are prominent factors in discrete FA are to be defined. In most of the combinatorial problems like scheduling the distance does not mean physical distance so proper attention is to be paid. Some of these metrics used for distance are time delay, time difference, Hamming distance, edit distance or Jaccard similarity. Solutions defining the neighborhood will have an impact on overall performance of implementation. Hence, the algorithms related to discrete Meta heuristic acts as a key role in the activities related to current research.

\section{FIREFLY ALGORITHMS IN APPLICATIONS}

Firefly algorithm mapped to the diversified modern day applications, [6, 10, 24, 42, 50, 25, and 26].Yang proposed in 2008, Nearly 810 papers are published, hence a handful of papers are hence putforth. FA has been used in image registration [58] by zhang and wu. Basing on time and optimality parameters of feature selection, The FA has produced optimal performance over the rest [7] in Banati and Bajaj. For the calculation of the least computation time for digital image compression [25, 26], FA showed the least which was disclosed by Horng et.al. Even in engineering design, the problems like highly non-linear, multimodal design problems have been efficiently solved, Gandomi et.al [20] and Azad [5] when it comes to radio communications optimization of antenna design, The FA has showed better performance than ABC[10], Mahanti[8], Chatterjee et.al. According to zaman and Matin FA can outperform PSO and achieved best results globally [57].

Some of the scheduling problems [42] of NP- hard have been effectively solved by the discrete version of FA developed by Sayadi et.al. Thegefficiency of FA has been clearly demonstrated in test problems like multi-objective load dispatch [6, 49, 52 ] to name a few. The value loading effect in non-convex economic dispatch problems got best result with FA over the others [54]. Yan et.al, economic load dispatch problems with reduced power losses have been solved using FA by Swarnkar [45\$.0 The scheduling in traveling salesman problems hazebeen solved promisingly by FA [39, 29, 56]. Jati and Suyanto, solved the traveling salesman problem using discrete FA. In these areas FA showed its efficiency. A version of Cuckoo search algorithm has been used to solve Traveling Salesman Problem that was elaborated by Ouaarab A, Ahiod B, Yang XS [38]. FA has demonstrated well for querying systems a study done by Kwiecian and Filipowicz [31].FA has proved well in finding solutions for mixed integer programming and local dispatch problems [9, 54].

We can find the applications of FA in classification and clustering with a good performance metric [43, 40]. The performance of FA is evaluated and concluded that FA can be efficiently used for clustering [43]. It has been performed by comparing with 11 different algorithms Senthilnath et.al. The FA is at the highest position in this evaluation. In neural network (NN) [36] FA has been applied for training [36]. The efficiency of FA is optimum in dynamic environments by Farahani et.al [15, 16] and Abshouri et.al [1]

The problems related to ISO spectral spring-mass systems has been solved effectively [13] by FA, Dutta et al. The forecasting of prices in the stock market [30] using support vector regression with the chaos-based FA has been presented by Kazem et.al.

The failure correction of antenna had been flexibly and effectively [23] studied by Grewal et.al. The effectiveness and optimal performance for generating individual test sequence in software testing has been showed by Srivastava et.al [44].

Firefly algorithm for function optimization and modelling to generate alternatives with the firefly algorithm in real world has been effectively elaborated by Imanirad, Xin-She Yang 2 and Julian Scott Yeomans[28]

\section{CONCLUSION}

Firefly Algorithm, Is it Efficient? The emerging new ideas are expanding the literature of FA and FA can outperform a lot more algorithms.

To answer the query "Why FA is efficient", it can be viewed in other dimensions. Swarm intelligent - based algorithms like FA have a lot more advantages.
Published By:

Blue Eyes Intelligence Engineering

\& Sciences Publication 
This algorithm by its design divides the group automatically and it has the ability to deal with multimodality. Firstly, FA property attraction and attractiveness which automatically subdivides the population into groups and each group can swarm around the small regions called as local regions. From each local optima that can be grouped to attain global optima as a best solution. Secondly, the subdivision grouping will generate local optima simultaneously. So, with an average distance between the groups the population can be subdivided. Where the average distance is $1 / \sqrt{\gamma}$.

The complete population will not sub divide, if ' $\gamma$ ' $=0$. By its design nature, The FA can be applied or suitable to non-linear, multi modal problems. The algorithm randomness can be fine turned as iterations proceed and the continuous problems, classifications, clustering and optimization are flexibly handled by Firefly algorithm.

Ultimately FA has

$>$ Subdivision of the population into groups
automatically
$>\begin{aligned} & \text { Ability to deal with optimization in } \\ & \text { multimode }\end{aligned}$
$>\begin{aligned} & \text { Optimal ergodicity and diversity in the } \\ & \text { solutions }\end{aligned}$

These factors make FA unique, effective and an efficient algorithm

\section{REFERENCES}

1. Abshouri AA, Meybodi MR, Bakhtiary A. "New firefly algorithm based on multiswarm and learning automata in dynamic environments". In: Third international conference on signal processing systems (ICSPS 2011), August 27-28, Yantai, China; 2011. p. 73-7.

2. Abdullah A, Deris S, Anwar S, Arjunan SNV. "An evolutionary firefly algorithm for the estimation of nonlinear biological model parameters". PLoS One 2013; 8(3):e56310.

3. AmiriB,Hossain L, Crawford JW,WigandRT. "Community detection in complex networks: Multi-objective enhanced firefly algorithm". Knowl Based Syst 2013; 46 (1):1-1.

4. Arsuaga-Rios M, Vega-Rodriguez MA. "Multi-objective firefly algorithm for energy optimization in grid environments. Swarm Intelligence". Lect Notes Comput Sci 2012;7461: 350-1.

5. Azad SK, Azad SK. "Optimum design of structures using an improved firefly algorithm”. Int .J. Optim Civil Eng 2011;1(2):327-40.

6. Apostolopoulos T, Vlachos A. "Application of the firefly algorithm for solving the economic emissions load dispatch problem”. Int. J.Combin 2011[Article ID 523806].

7. Banati H, Bajaj M. "Firefly-based feature selection approach". Int. J. Comput Sci 2011; 8 (2):473-80.

8. Basu B, Mahanti GK. "Firefly and artificial bee colony algorithm for synthesis of scanned and broadside linear array antenna". Prog Electromagnet Res B 2011; 32 (1):169-90.

9. Chandrasekaran K, Simon SP. "Network and reliability constrained unit commitment problem using binary real coded firefly algorithm". Int J Electr Power Energy Syst 2012; 42 (1):921-32.

10. Chatterjee A, Mahanti GK, Chatterjee A. "Design of a fully digital controlled reconfigurable switched beam concentric ring array antenna using firefly and particle swarm optimization algorithm". Prog Elelectro magnet Res B 2012; 36(1):113-31.

11. Coelho LS, de Andrade Bernert DL, Mariani VC. "A chaotic firefly algorithm applied to reliability-redundancy optimization”. In: 2011 IEEE Congress on Evolutionary Computation (CEC'11); 2011. p. 517-21.

12. Coelho LS, Mariani VC. Firefly algorithm approach based on chaotic Tinker bell map applied to multivariable PID controller tuning. Comput Math Appl 2012; 64 (8):2371-82. DOI: 10.1016/j.camwa.2012.05.00.
13. Rajdeep Dutta, Ranjan Ganguli, and V. Mani, Exploring isospectral spring-mass systems with firefly algorithm 467. Proceedings of the Royal Society A: Mathematical, Physical and Engineering Sciences, 467(2135): 3222-40, http://doi.org/10.1098/rspa.2011.0119.

14. Implementation of a discrete firefly algorithm for the QAP problem within the sage framework. Karel Durkota. Bachelor Thesis, Czech Technical University (2011), http://cyber.felk.cvut.cz/research/theses/papers/189.pdf, 2013 [accessed 23.05.13].

15. SM Farahani, AA Abshouri, B Nasiri, MR Meybodi, Some hybrid models to improve firefly Algorithm performance, International Journal of Artificial Intelligence 8 (12), 97-117.

16. Farahani SM, Nasiri B, Meybodi MR. A multiswarm based firefly algorithm in dynamic Environments. In: Third international conference on signal processing systems (ICSPS 2011), August 27-28, Yantai, China; 2011. Vol. 3, pp. 68-72(2011).

17. Farahani SM, Abshouri AA, Basiri B, Meybodi MR, A Gaussian firefly algorithm. Int. J. Mach Learn Comput 2011; 1(5):448-53.

18. I. Fister Jr., X.-S. Yang, I. Fister, J. Brest. Memetic firefly algorithm for combinatorial optimization in Bio inspired Optimization Methods and their Applications (BIOMA 2012), B. Filipic and J. Silc, Eds. Jozef Stefan Institute, Ljubljana, Slovenia, 24-25 May 2012, p. 75-86.

19. A comprehensive review of firefly algorithms Citation Data Swarm and Evolutionary Computation, Swarm Evol Comput 2013, ISSN: 2210-6502, Vol. 13, 14 Page: https://doi.org/10.1016/j.swevo.2013.06.001.

20. Gandomi, A.H., Yang, X.S. and Alavi, A.H. (2011) Mixed Variable Structural Optimization Using Firefly Algorithm. Computers and Structures, $\quad 89$ 2325-2336. http://dx.doi.org/10.1016/j.compstruc.2011.08.002.

21. A.H. Gandomi, X.-S. Yang, S. Talatahari, A.H. Alavi, Firefly algorithm with chaos, Communications in Nonlinear Science and Numerical Simulation, Volume 18, Issue 1, 2013, Pages 89-98, ISSN 1007-5704,https://doi.org/10.1016/j.cnsns.2012.06.009.(http://www.s ciencedirect.com/science/article/pii/S1007570412002717)

22. Giannakouris G., Vassiliadis V., Dounias G. (2010) Experimental Study on a Hybrid Nature-Inspired Algorithm for Financial Portfolio Optimization. In: Konstantopoulos S.,Perantonis S., Karkaletsis V., Spyropoulos C.D., Vouros G. (Eds) Artificial Intelligence: Theories, Models and Applications. SETN 2010. Lecture Notes in Computer Science, vol.040. Springer, Berlin, Heidelberg, 2010. p. 101-111.doi https://doi.org/10.1007/978-3-642-12842-4_14.

23. N. S. Grewal, M. Rattan, and M. S. Patterh, A Linear Antenna Array Failure Correction Using Firefly Algorithm, progress In Electromagnetics Research M, Vol. 27, 241-254, 2012.doi. 10.2528/PIERM12101903.

24. Hassanzadeh T, Vojodi H, Moghadam AME. An image segmentation approach based on Maximum variance intra-cluster method and firefly algorithm. In: Proceedings of the Seventh International Conference on natural computation (ICNC 2011); 2011. p. 1817-21.

25. Horng MH, Lee YX, Lee MC, Liou RJ. Firefly metaheuristic algorithm for training the radial basis function network for data classification and disease diagnosis. In: Parpinelli R, Lopes HS, editors. Theory and new applications of swarm intelligence; 2012. p. 115-32.

26. Horng, Ming-Huwi. (2012). Horng, M.H.: Vector Quantization Using the Firefly Algorithm for Image Compression. Expert Syst. Appl. 39(1), 1078-1091. Expert Systems with Applications: An International Journal.39.1078-1091.10.1016/j.eswa.2011.07.108.

27. Horng $\mathrm{MH}$, Liou RJ. Multilevel minimum cross-entropy threshold selection based on the firefly algorithm. Expert Syst. Appl. 2011; 38(9):14805-11.

28. Imanirad R, Yang XS, Yeomans JS. Modeling-to-generate-alternatives via the firefly algorithm. J. Appl Oper Res 2013; 5(1):14-21.

29. Jati GK, Suyanto S. Evolutionary discrete firefly algorithm for traveling salesman problem. ICAIS 2011, Lecture notes in artificial intelligence (LNAI 6943) 2011; 2011:393-403.

30. Kazem A, Sharifi E, Hussain FK, Saberi M, Khadeer O. "Support vector regression with chaos-based firefly algorithm for stock market price forecasting". Appl Soft Comput 2013; 13(2):947-58.

31. Kwiecia'n J, Filipowicz B. Firefly algorithm in optimization of queuing systems. Bull Pol Acad Sci Tech Sci 2012; 60(2):363-8.

32. Lewis SM, Cratsley CK. Flash signal evolution, mate choice and predation in fireflies. Ann Rev Entomol 2008; 53(2):293-321. doi: 10.1146/annurev.ento.53.103106.093346

\section{Published By:}

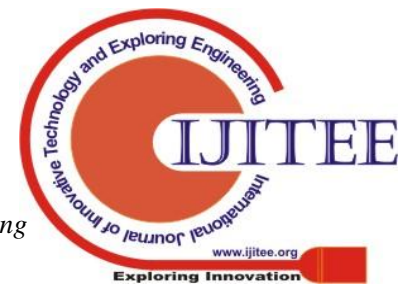


33. Li HM, Ye CM. Firefly algorithm on multi-objective optimization of production scheduling System. Adv. Mech. Eng. Appl. 2012; 3(1):258-62.

34. Luz EFP, Campos Velho HF, Becceneri JC. Firefly algorithm with predation: a parallel implementation applied to inverse heat conduction problem. In: Proceedings of 10th world congress on computational mechanics (WCCM 2012; conference presentation); 2012.

35. Marichelvam MK, Prabaharan T, Yang XS. A discrete firefly algorithm for the multi objective hybrid flow shop scheduling problems. IEEE Transactions on Evolutionary Computation Volume.18, Issue2, April2014.http://ieeexplore.ieee.org/xpl/articleDetails.jsp?arnumber= 6412790.

36. Nandy S, Sarkar PP, Das A. Analysis of nature-inspired firefly algorithm-based back propagation neural network training. Int. J. Comput Appl 2012; 43(22):8-16.

37. Nasiri B, Meybodi MR. Speciation-based firefly algorithm for optimization in dynamic environments. Int. J. Artificial Intelligence 2012; 8(S12):118-32.

38. Ouaarab A, Ahiod B, Yang XS. Discrete cuckoo search algorithm for the travelling salesman problem. Neural computing and applications, 2013.

http://link.springer.com/article/10.1007\%2Fs00521-013-1402-2.

39. Palit S, Sinha S, Molla M, Khanra A, Kule M. A cryptanalytic attack on the knapsack cryptosystem using binary firefly algorithm. In: Second international conference on computer and communication technology (ICCCT), 15-17, Sep.2011, p. 428-32.

40. Rajini, A., David, V.K. A hybrid metaheuristic algorithm for classification using micro array Data. Int. J. Sci. Eng. Res 2012; 3(2):1-9.

41. Rampriya B, Mahadevan K, Kannan S. Unit commitment in deregulated power system using Lagrangian firefly algorithm. In: Proceedings of IEEE international conference on communication control and computing technologies (ICCCCT2010); 2010. p. 389-93, doi.10.1109/ICCCCT.2010.5670583.

42. Sayadi MK, Ramezanian R, and Ghaffari-Nasab N. A discrete firefly meta-heuristic with local search for make span minimization in permutation flow shop scheduling problems. International Journal of Industrial Engineering Computations, 2010; 1(1): 1-10, doi. 10.5267/j.ijiec.2010.01.001

43. J. Senthilnath, S.N. Omkar, V. Mani, Clustering using firefly algorithm: Performance study, Swarm and Evolutionary Computation, Vol. 1, I. 3, 2011, P. 164-171, ISSN 2210-6502, https://doi.org/10.1016/j.swevo.2011.06.003.http://www.sciencedirec t.com/science/article/pii/S2210650211000265.

44. Srivastava PR, Mallikarjun B, Yang XS. Optimal test sequence generation using firefly algorithm. Swarm Evol Comput 2013; 8(1):44-53.

45. Swarnkar KK. Economic load dispatch problem with reduce power losses using firefly algorithm. J. Adv. Comput. Sci. Tech. 2012; 1(2):42-56.

46. Tang R, Fong S, Yang XS, Deb S. Integrating nature-inspired optimization algorithm to K-means clustering. In: The seventh international conference on digital information management (ICDIM2012), 22-24 August. Macau: IEEE Publications; 2012. p. 116-23.

47. Wang GG, Guo LH, Duan H, Liu L, Wang HQ. A modified firefly algorithm for UCAV path planning. Int. J. Hybrid Inform Tech. 2012; 5(3):123-44.

48. Yang XS. Nature-inspired metaheuristic algorithms. 1st ed. Frome, UK: Luniver Press; 2008.

49. X.-S. Yang, Firefly algorithms for multimodal optimization, in: Stochastic Algorithms: Foundations and Applications, SAGA 2009 Lecture Notes in Computer Sciences, Vol. 5792, pp. 169-178 (2009).

50. Yang XS. Firefly algorithm, stochastic test functions and design optimization. Int. J. Bio-Inspired Computation 2010; Vol. 2, No 2:78-84.

51. Yang XS. Chaos-enhanced firefly algorithm with automatic parameter tuning. Int. J. Swarm Intell. Research 2012; 2(4):1-11.october.

52. Yang XS. Swarm-based metaheuristic algorithms and no-free-lunch theorems. In: Parpinelli R, Lopes HS, editors. Theory and new applications of swarm intelligence. In tech Open Science; 2012. pp. 1-16.doi: https://doi.org/10.5772/30852.

53. X. S. Yang, Multi objective firefly algorithm for continuous optimization, Engineering with Computers, Vol. 29, No. 2, pp. 175-184 (2013).

54. Yang XS, Hosseini SSS, Gandomi AH. Firefly algorithm for solving non-convex economic dispatch problems with valve loading effect.
Appl. Soft Comput. 2012; vol. 12, No. 3, pp.1180-86. doi: 10.1016/j.asoc.2011.09.017.

55. Yan X, Zhu YL, Wu JW, Chen $\mathrm{H}$. An improved firefly algorithm with adaptive strategies. Adv. Sci. Lett.. 2012;16(1):249-54.

56. Yousif A, Abdullah AH, Nor SM, AbdelAziz A. Scheduling jobs on grid computing using firefly algorithm. J. Theory Appl. Inform. Technol. 2011; Vol. 33, No. 2, pp.155-64.

57. Zaman MA, Matin MA. Non- uniformly spaced linear antenna array design using firefly algorithm. Int. J. Microwave Science and Technology, vol. 2012 [Article ID: 256759]. Doi:10.1155/2012/256759.

58. Zhang YD,Wu LN. A novel method for rigid image registration based on firefly algorithm. Int. J. Res. Rev. Soft. Intell. Comput. 2012; Vol. 2 , No.2, pp.141-46.

\section{AUTHORS PROFILE}

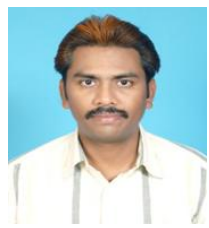

Mr. N. Venkata Ramana Gupta , Research Scholar, Department of Computer science and Engineering, Acharya Nagarjuna University, Nagarjuna Nagar. He is also working as Assistant Professor in Department of Computer Science and Engineering Prasad V. Potluri Siddhartha Institute of Technology (Autonomous) Vijayawada, Andhra Pradesh. He obtained his Masters in (Computer Science and Technology with specialization in computer Networks) from Andhra University College of Engineering, Visakhapatnam, AP.His areas of interest are Computer Networks, Distributed Systems. He is member of ACM and Life Member of ISTE.

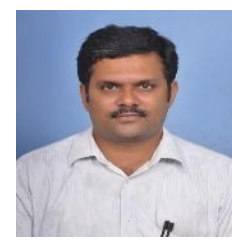

Dr. Ramesh Makala is an associate professor in the Department of Information Technology at RVR \& JC College of Engineering, Guntur, Andhra Pradesh, India. He received his Ph.D. in Computer Science from Acharya Nagarjuna University in 2013 and M.Tech. from Andhra University in 2004 and MCA from Andhra University in 1996. His research interests span both Image Processing and Information Security. Much of his work has been on security and data compression. He has published different novel works in IEEE Conferences and Scopus Indexed journals. He is in charge of the Research Center in the department. He also guides students who are working on Machine Learning and Big Data Analytics problems. 